\title{
Hemispheric specialization in selective attention and short-term memory: a fine-coarse model of left- and right-ear disadvantages
}

\author{
John E. Marsh',2, Lea K. Pilgrim ${ }^{1}$ and Patrik Sörqvist ${ }^{2,3}$ * \\ ' School of Psychology, University of Central Lancashire, Preston, Lancashire, UK \\ ${ }^{2}$ Department of Building, Energy, and Environmental Engineering, University of Gävle, Gävle, Sweden \\ ${ }^{3}$ Linnaeus Centre HEAD, Swedish Institute for Disability Research, Linköping University, Linköping, Sweden
}

\section{Edited by:}

Marco Hirnstein, University of

Bergen, Norway

\section{Reviewed by:}

Kristiina Kompus, University of Bergen, Norway

Gina M. Grimshaw, Victoria University of Wellington, New Zealand

\section{${ }^{*}$ Correspondence:}

Patrik Sörqvist, Department of Building, Energy and Environmental Engineering, University of Gävle, SE-801 76 Gävle, Sweden e-mail: patrik.sorqvist@hig.se

\begin{abstract}
Serial short-term memory is impaired by irrelevant sound, particularly when the sound changes acoustically. This acoustic effect is larger when the sound is presented to the left compared to the right ear (a left-ear disadvantage). Serial memory appears relatively insensitive to distraction from the semantic properties of a background sound. In contrast, short-term free recall of semantic-category exemplars is impaired by the semantic properties of background speech and is relatively insensitive to the sound's acoustic properties. This semantic effect is larger when the sound is presented to the right compared to the left ear (a right-ear disadvantage). In this paper, we outline a speculative neurocognitive fine-coarse model of these hemispheric differences in relation to short-term memory and selective attention, and explicate empirical directions in which this model can be critically evaluated.
\end{abstract}

Keywords: ear-advantage, hemispheric asymmetry, distraction, short-term memory, left-ear disadvantage, right-ear disadvantage
One way in which our understanding of hemispheric specialization has been advanced is through the study of auditory processing (Cherry, 1953; Broadbent, 1958; Hugdahl, 2003; Hugdahl et al., 2009). Specifically, the combination of weaker ipsilateral pathways and stronger contralateral pathways within the auditory system results in the contralateral processing of sound. Input to the left ear, for example, has privileged access to the right hemisphere (RH), whereas input to the right ear has privileged access to the left hemisphere (LH). Sound, such as speech, is therefore predominately processed by the opposite hemisphere to its presentation source. These hemispheric differences result in the right-ear advantage in identifying or shadowing linguistic target-stimuli that are presented to the right-ear/LH (Kimura, 1961, 1967; Broadbent and Gregory, 1964; Studdert-Kennedy and Shankweiler, 1970) and the left-ear advantage for the processing of non-linguistic sound presented to the left-ear/RH (Tervaniemi and Hugdahl, 2003; Poeppel etal., 2004), especially with binaural sound presentation, although ear-advantages with monaural presentation have also been shown (Bradshaw etal., 1981). In this article, we review existing work on how this hemispheric asymmetry influences selective attention and short-term memory in the context of cross-modal auditory distraction.

\section{CROSS-MODAL DISTRACTION THE IRRELEVANT SOUND EFFECT (AND RIGHT HEMISPHERE PROCESSING)}

The irrelevant sound effect refers to the observation that shortterm memory for the correct serial order of a set of sequentially presented visual items (visual-verbal serial recall) is disrupted by the mere presence of background sound. Despite explicit instruction to ignore the sound, error rates can increase by up to $50 \%$ (Ellermeier and Zimmer, 1997). Two pre-requisites for irrelevant sound to produce substantial disruption are, first, that the focal task involves serial rehearsal of the to-be-recalled (TBR) items (Beaman and Jones, 1997), and second, that the irrelevant sound demonstrates appreciable acoustic variation from one sound element to the next (Jones and Macken, 1993). For example, if participants are required to maintain the serial order of TBR items, auditory changing-state sequences (e.g., "a b a b a b a") are typically more disruptive than steady-state sound sequences (e.g., "a a a a a a a"), the changing-state effect. However, if participants are required to identify which member of a well-known set (e.g., Weekdays) that is not presented - the missing-item task - the changing-state effect does not emerge (Beaman and Jones, 1997). The theoretical reason for this is that the missing-item task does not require seriation of the TBR items, and so there is no conflict between the order information in the changing-state sequence and the processes that are required to fulfill the task. The combination of these two prerequisites suggests that the changing-state effect is a function of the similarity between two sets of order processes: The deliberate processing of the order of the TBR items and the involuntary processing of the order between successive and perceptually discrete sound events (for a review, see Jones et al., 2010).

It does not matter whether the changing-state sequence consists of speech utterances or pure tones (e.g., Jones and Macken, 1993), the magnitude of disruption is rather a function of the sound's acoustic variation (Jones et al., 2000), which suggests that the sound's phonological and semantic content plays little if any role, although this is still the subject of debate (e.g., Bell et al., 
2011). Item identity typically plays a more subservient role than acoustic variation (Jones et al., 2010), but serial recall of visual digits is more greatly impaired by irrelevant digits than irrelevant consonants if the order of the irrelevant digits is different (i.e., incongruent) to the order of the TBR digits, but not when it is similar (congruent; Hughes and Jones, 2005; Bell et al., 2011).

The changing-state effect has been used as an analytic tool to study hemispheric biases for passive processing of irrelevant sound. For example, Hadlington et al. (2004) found that speech utterances (Experiments $1 \mathrm{a}$ and $2 \mathrm{a}$ ) and sine wave tones (Experiments $1 \mathrm{~b}$ and $2 \mathrm{~b}$ ) impair serial memory to a greater extent when presented to the left-ear-only, relative to right-ear-only presentation and presentation to both ears. This left-ear disadvantage was later replicated in the context of a mental arithmetic task (cf. Banbury and Berry, 1998), but was not found in the context of a missing-item task (Hadlington et al., 2006). Moreover, the leftear disadvantage was greater in magnitude when the irrelevant sequence conveyed acoustic variation, such as pitch changes, and varied inter-stimulus intervals, but it was absent when the sound stream contained little acoustic variation (such as a repetition of a single utterance). Collectively, serial short-term memory is more impaired from sound with a left-ear source, and it does not matter if that sound has lexico-semantic content or not.

These findings cohere nicely with the notion that the RH plays a prominent role in the obligatory processing of the acoustic features rather than the item identity/content within the irrelevant sound streams (Zatorre et al., 1994; Grimshaw et al., 2003; Poeppel et al., 2004): The RH specialization for processing serial information turns into a disadvantage when sound is to-be-ignored and the focal task also requires seriation.

\section{ITEM BASED DISTRACTION (AND LEFT HEMISPHERE PROCESSING)}

As discussed above, acoustic variation appears to interfere selectively with serial memory in the $\mathrm{RH}$, due to a conflict between deliberate order processes and an automatic analysis of acoustic change in the unattended sound. Both behavioral and neuroimaging studies propose that order and item information are supported by separate cognitive representations (for a review, see Marshuetz, 2005), which suggests that background sound could selectively impair item memory, just as it selectively impairs serial memory in the RH. This is the question we turn to next.

The LH appears to dominate language/semantic processing. For example, little lexical-semantic analysis of deliberately ignored speech is thought to take place in the RH (Beaman et al., 2007) and the LH responds to lexical-semantic information of auditory word stimuli (Zahn et al., 2000). Moreover, memory for verbal material is LH localized (e.g., Smith et al., 1996; Baddeley, 2003) and the right-ear advantage in dichotic listening (e.g., Hugdahl et al., 2009) supports privileged linguistic processing in the LH. Taken together, in the context of tasks that require semantic processing, which predominantly depend on the LH, background speech might actually be more disruptive when presented to the right ear. This hypothesis has recently received some support (Sörqvist et al., 2010).

Sörqvist et al. (2010) used a paradigm wherein TBR visual lists comprise exemplars that are members of the same semantic category (e.g., Fruit). To-be-ignored spoken words that are taken from the same semantic category as the TBR items (e.g., other Fruit) produce greater disruption to free recall than to-be-ignored words from a different semantic category (e.g., Tools): the betweensequence semantic similarity effect (Marsh et al., 2008). This effect is indexed as fewer correct recalls of visual-targets and greater false recall (e.g., of words that were spoken in the background). The between-sequence semantic similarity effect is found when speech is presented to the right-ear/LH but not when it is presented to the left-ear/RH (Sörqvist et al., 2010). Importantly, this right-ear disadvantage is only found when the task is to recall the items in free order (Experiments 1 and 3), not when they must be recalled in order of presentation (Experiment 2).

Thus, task requirement appears to determine when a left-ear or a right-ear disadvantage is found. Verbal item memory, localized to the LH, is more impaired when task-irrelevant linguistic information is presented to the right-ear/LH, whereas serial order memory, predominantly localized to the $\mathrm{RH}$, is more impaired by acoustically varying sound presented to the left-ear/RH. Interestingly, the ear-disadvantages have been shown in the context of monaural sound presentation, whereas the ear-advantages are typically found with binaural presentation. A right-ear advantage is found with monaural presentation, however, when several sound streams are presented simultaneously to the same ear, and there is a need to resolve stimulus competition (Bradshaw et al., 1981). Taken together with the cross-modal effects, the ear asymmetries in monaural presentation may arise because of the competition between processing streams.

\section{False recall}

In the context of free recall, spoken words that are related (e.g., Tools) to the TBR items (e.g., Tools) typically produce more false recall (of items that belong to the target category, but were not part of the target list) than unrelated spoken words (e.g., Occupations; Marsh et al., 2008). This effect is greater for right-ear/LH presentation (Sörqvist et al., 2010). Initially, these results appear consistent with the model offered by Beaman et al. (2007) wherein it is assumed that right-ear input increases the capacity of speech to interfere with the semantic processes in the LH, whereas this capacity is attenuated for left-ear input. However, Sörqvist et al. (2010) found that unrelated speech presented to the left-ear/RH resulted in more false recall than unrelated speech presented to the right-ear/LH (i.e., a left-ear disadvantage for the effect of unrelated speech on false recall). Moreover, despite generation of fewer intrusions with unrelated speech presented to the right-ear/LH, those that emerged were generally greater in output-dominance (e.g., DOG is a more dominant member than LIZARD of the category "four-legged animal"). The finding that unrelated speech presented to the left-ear/RH has systematic effects on false recalls suggests some lexical-semantic processing of irrelevant speech within the RH.

\section{UNDERSTANDING THE PATTERN OF INTRUSIONS ACROSS THE HEMISPHERES}

Hemispheric asymmetries in (attended) semantic processing are well documented. For example, Beeman and colleagues (Beeman et al., 1994; Beeman and Chiarello, 1998; Bowden and Beeman, 1998; Beeman and Bowden, 2000; Bowden and Jung-Beeman, 
2003) suggest that the LH is particularly attuned to fine processing, activating a restricted semantic network comprising of a small number of closely related concepts. In contrast, the RH specializes in coarse processing (Tervaniemi and Hugdahl, 2003) activating a widespread, diffuse array of associates.

This fine-coarse processing mechanism is supported by evidence from semantic priming. Greater summation priming from three weakly-related, centrally-presented, prime words (e.g., footcry-glass) is found when the target word (e.g., cut) is presented to the left visual-field/RH as opposed to the right visual-field/LH (Beeman et al., 1994). In contrast, directly related primes (e.g., scissors) show greater priming than summation primes when targets are presented in the right visual-field/LH. The idea is that the RH weakly activates large semantic fields, which overlap, and therefore, although each semantic field is only weakly activated, this overlap allows the weakly related concepts to activate more strongly, reaching threshold. In contrast, the LH strongly activates narrow semantic fields, activating only dominant meanings, or meanings that are most relevant to the immediate context.

The novel approach that we take here is to attempt to explain how Beeman et al.'s (1994) fine-coarse model can account for the findings that (a) an unrelated stream of words presented to the right-ear/LH suppresses false recall, and that (b) the intrusions produced when unrelated words are presented to the right-ear/LH are greater in output-dominance (Sörqvist et al., 2010). One possible explanation for these findings, that concerns false recalls, can be found in relation to how hemispheric differences in semantic activation influences selection of candidates for recall.

Semantic activation across the hemispheres is defined in terms of: speed, strength, and breadth. In the LH, semantic activation quickly focuses in on a narrow semantic field of strongly activated items relevant to the current task. In contrast, the pattern in the $\mathrm{RH}$ is more diffuse and weak, activating a broad semantic field of both more and less relevant related items. These different patterns of activation across the hemispheres (LH: quick, small, strong versus RH: slow, broad, weak), are likely to result in a different level of false recall. We expand on this point below.

\section{LEFT HEMISPHERE PRESENTATION Unattended related items}

Strong activation quickly narrows down to focus on the cohort of relevant items (i.e., the TBR items). Some of the unattended related items would also fall within this narrow semantic field (Figure 1, Panel 1a). Connectivity between all these related items would likely boost levels of activation within the entire cohort. Furthermore, unattended items that are activated are likely to be a) LH: related

Panel 1

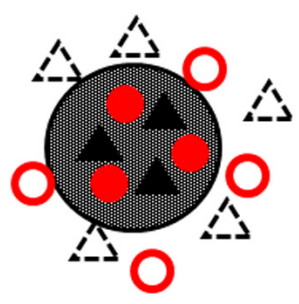

a) $\mathrm{RH}$ : related

Panel 2

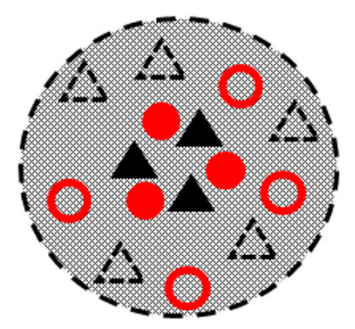

b) LH: unrelated
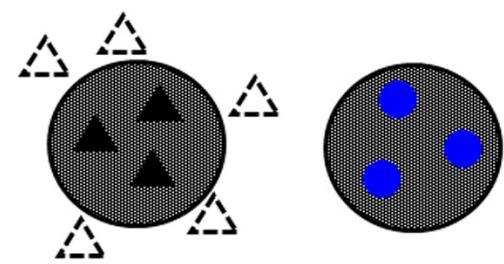

b) $\mathrm{RH}$ : unrelated

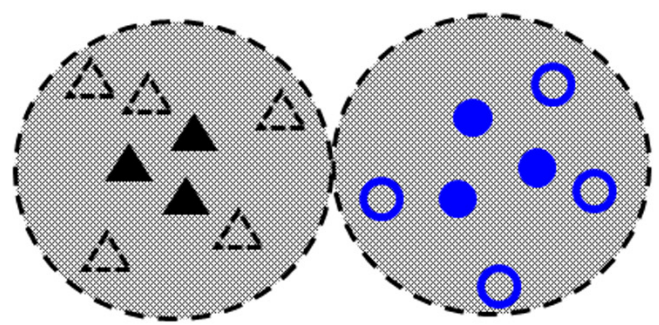
TBR (attended) items
Non-presented items related to TBR items
Related unattended dominant items
Related unattended non-dominant items
Unrelated unattended dominant items
Unrelated unattended non-dominant items

FIGURE 1 |The figure shows the fine-coarse model of semantic activation when TBR and unattended items are presented to the right-ear/LH (Panel 1) or left-ear/RH (Panel 2), and when the unattended items are either semantically related (a) or sematically unrelated (b). 
the ones that are most closely related to the TBR items. In contrast, more distantly related items are likely to be outside this core semantic network and, thus, would not benefit from the strong activation and interconnectivity between items. This is likely to result in high levels of intrusion from closely related (dominant) items but with little interference from (and hence false recall of) distantly related (non-dominant) items.

\section{Unattended unrelated items}

The TBR and unattended unrelated items activate two separate semantic networks (Figure 1, Panel 1b). However, unlike the related condition, there would be no connectivity between TBR and unattended items and thus little interference. As with the unattended related items, false recall would likely be confined to dominant items closley related to the TBR items.

\section{RIGHT HEMISPHERE PRESENTATION Unattended related items}

The RH weakly activates a broad, diffuse semantic network encompassing both TBR and unattended related items. Although intrusions are less likely than in the LH (due to weaker activation), the wide network of interconnected related items suggests that some unattended items are likely to reach a threshold where false recall is possible. Due to the broad semantic network activated, these intrusions would be equally likely from either closely related (dominant) or weakly related (non-dominant) items (Figure 1, Panel 2a). In addition, the diffuse activation makes it possible that related, but non-presented items, are also activated ${ }^{1}$. However, because of the strong competition from the mutally activating TBR and unattended items, non-presented items are unlikely to reach threshold for intrusion.

\section{Unattended unrelated items}

The TBR and unattended unrelated items activate two separate semantic networks (see Figure 1 Panel 2b). Thus, unlike the unattended related items, the unattended unrelated items do not benefit from mutual activation via the TBR items. This would result in them having less chance of reaching threshold, as they receive no boost from interconnectivity with the TBR items. However, intrusions in the unattended unrelated condition would still be more likely in the RH than the LH, because the broad semantic network allows a greater connectivity between cohort members than in the $\mathrm{LH}$, resulting in increased levels of activation for some items within the cohort. As in the unattended related condition, intrusions would be equally likely from either closely related (dominant) or weakly related (non-dominant) items. Finally, intrusions from non-presented items that are related to the TBR items may be slightly higher than in the unattended related condition, because they benefit from connnectivity with the TBR items, but do not suffer from additional competition from the unattended items.

\section{EXTENSIONS AND PREDICTIONS OF THE MODEL}

The fine-coarse model of hemispheric specialization supposes that the retrieval information from semantic memory (a process that underpins short-term memory for identity) will be vulnerable to

\footnotetext{
${ }^{1}$ In contrast, in the LH it is unlikely that non-presented items would be activated
} due to the narrow semantic field activated (see in Figure 1 Panel 1). disruption via meaningful speech presented to the right-ear/LH whereas the process of serial rehearsal (a process that underpins short-term memory for order) will be more impaired by acoustically variable sound presented to the left-ear/RH. Moreover, the model suggests that the ways in which background speech promotes false recall will depend on the semantic relation between targets and distracters and the dominance of the distracters. In general, the empirical findings presented here are consistent with the fine-coarse model.

The concept of hemispheric asymmetry in processing suggested by the fine-coarse model can be theoretically useful in informing the debate between interference-by-process (Jones and Tremblay, 2000; Marsh et al., 2009) and interference-by-content (Salamé and Baddeley, 1982, 1986; Neath, 2000) accounts of auditory distraction within short-term memory. The findings reviewed here are at odds with the interference-by-content approach whereby the irrelevant sound effect is viewed as a function of the similarity in identity between the TBR and irrelevant items. In contrast, the findings with by-ear presentation harmonize with the more dynamic interference-by-process account according to which the type of distraction that takes place (item or order based distraction) does not depend on the materials of the focal task but on the nature of the cognitive operations that are carried out to process that material. Here, we outline ways in which the fine-coarse model can be used to further explore this distinction between item and order based distraction.

\section{FREE RECALL}

One way to test the predictions of the fine-coarse model, as outlined, is through manipulating the output-dominance of the unrelated speech within free recall. Low output-dominant items that are weakly representative of their category should result in more activation - and hence promote more false recalls when presented to the left-ear/RH in comparison with presentation to the right-ear/LH. A smaller by-ear effect should be found for the presentation of unrelated speech that conveys high output-dominant category members.

\section{SERIAL RECALL}

As noted, similarity in item identity between target and distracters can play a role in disruption of serial recall (Hughes and Jones, 2005). This can be further explored through manipulating the size of the TBR item set. For example, letters come from a wider set (26 in English) than digits (0-9) and thus the burden on item memory can be greater with letters. By-ear presentation could yield some clues as to whether some variants of the serial recall task simply tap into item-based effects. Specifically, the role of the RH (and therefore the left-ear disadvantage) should be much reduced (and possibly even turn into a right-ear disadvantage) when the serial recall task comprises a larger set (e.g., 8 of 26 items presented on any given trial). A further extension along these lines would be to investigate the role of individual differences. Individual differences in working memory capacity are unrelated to the magnitude of the changing-state effect (Sörqvist et al., 2013), but related to the ability to resist attention capture (Sörqvist, 2010) and to semantic effects (Beaman, 2004). As there are also substantial individual differences in ear-advantages (Hugdahl, 2000), perhaps the role 
for item-based disruption in the context of serial memory can be further explored by analyzing the relation between working memory capacity (WMC) and ear-disadvantages. Indeed, Beaman et al. (2007) suppose that WMC is associated with the capability to modify the activity or output from lexico-semantic analysis in the left superior temporal gyrus (STG).

\section{REFERENCES}

Baddeley, A. (2003). Working memory: looking back and looking forward. Nat. Rev. Neurosci. 4, 829-839. doi: 10.1038/nrn1201

Banbury, S. P., and Berry, D. C. (1998). Disruption of office-related tasks by speech and office noise. Br. J. Psychol. 89, 499-517. doi: 10.1111/j.20448295.1998.tb02699.x

Beaman, C. P. (2004). The irrelevant sound phenomenon revisited: what role for working memory capacity? J. Exp. Psychol. Learn. Mem. Cogn. 30, 1106-1118. doi: 10.1037/0278-7393.30.5.1106

Beaman, C. P., Bridges, A. M., and Scott, S. K. (2007). From dichotic listening to the irrelevant sound effect: a behavioral and neuroimaging analysis of the processing of unattended sound. Cortex 43, 124-134. doi: 10.1016/S0010-9452(08) 70450-7

Beeman, M. J., and Bowden, E. M. (2000). The right hemisphere maintains solution related activation for yet-to-be-solved problems. Mem. Cognit. 28, 1231-1241. doi: 10.3758/BF03211823

Beeman, M., and Chiarello, C. (eds). (1998). Right Hemisphere Language Comprehension: Perspectives from Cognitive Neuroscience. Mahwah, NJ: Lawrence Erlbaum Associates.

Beeman, M., Friedman, R. B., Grafman, J., Perez, E., Diamond, S., and Lindsay, M. B. (1994). Summation priming and coarse semantic coding in the right-hemisphere. J. Cogn. Neurosci. 6, 26-45. doi: 10.1162/jocn.1994.6.1.26

Beaman, C. P., and Jones, D. M. (1997). The role of serial order in the irrelevant speech effect: tests of the changing-state hypothesis. J. Exp. Psychol. Learn. Mem. Cogn. 23, 459-471. doi: 10.1037/0278-7393.23.2.459

Bell, R., Mund, I., and Buchner, A. (2011). Disruption of short-term memory by distractor speech: does content matter? Q. J. Exp. Psychol. 64, 146-168. doi: 10.1080/17470218.2010.483769

Bowden, E. M., and Beeman, M. J. (1998). Getting the right idea: semantic activation in the right hemisphere may help solve insight problems. Psychol. Sci. 9, 35-40. doi: $10.1111 / 1467-9280.00082$

Bowden, E. M., and Jung-Beeman, M. (2003). Aha! Insight experience correlates with solution activation in the right hemisphere. Psychon. Bull. Rev. 10, 730-737. doi: 10.3758/BF03196539

Bradshaw, J. L., Farrelly, J., and Taylor, M. J. (1981). Synonym and antonym pairs in the detection of dichotically and monaurally presented targets: competing monaural stimulation can generate a substantial right ear advantage. Acta Psychol. 47, 189-205. doi: 10.1016/0001-6918(81)90008-1

Broadbent, D. E. (1958). Perception and Communication. New York: Oxford University Press. doi: 10.1037/10037-000

Broadbent, D. E., and Gregory M. (1964). Stimulus set and response set: the alternation of attention. Q. J. Exp. Psychol. 16, 309-318. doi: $10.1080 / 17470216408416386$

Cherry, E. C. (1953). Some experiments on the recognition of speech, with one and with two ears. J. Acoust. Soc. Am. 25, 975-979. doi: 10.1121/1.1907229

Ellermeier, W., and Zimmer, K. (1997). Individual differences in susceptibility to the "irrelevant speech effect." J. Acoust. Soc. Am. 102, 2191-2199. doi: $10.1121 / 1.419596$

Grimshaw, G. M., Kwasny, K. M., Covell, E., and Johnson, R. A. (2003). The dynamic nature of language lateralisation: effects of lexical and prosodic factors. Neuropsychologia 41, 1008-1019. doi: 10.1016/S0028-3932(02)00315-9

Hadlington, L. J., Bridges, A. M., and Beaman, C. P. (2006). A left-ear disadvantage for the presentation of irrelevant sound: manipulations of task requirements and changing state. Brain Cogn. 61, 159-171. doi: 10.1016/j.bandc.2005.11.006

Hadlington, L. J., Bridges, A. M., and Darby, R. (2004). Auditory location in the irrelevant sound effect: the effects of presenting auditory stimuli to either the left ear, the right ear or both ears. Brain Cogn. 55, 547-557. doi: 10.1016/j.bandc.2004.04.001

Hugdahl, K. (2000). Lateralization of cognitive processes in the brain. Acta Psychol. 105, 211-235. doi: 10.1016/S0001-6918(00)00062-7
Hugdahl, K. (2003). "Dichotic listening in the study of auditory laterality," in The Asymmetrical Brain, eds K. Hugdhal and R. J. Davidson (Cambridge, MA: MIT Press).

Hugdahl, K., Westerhausen, R., Alho, K., Medvedev, S., Laine, M., and Hämäläinen, H. (2009). Attention and cognitive control: unfolding the dichotic listening story. Scand. J. Psychol. 50, 11-22. doi: 10.1111/j.1467-9450.2008.00676.x

Hughes, R. W., and Jones, D. M. (2005). The impact of order incongruence between a task-irrelevant auditory sequence and a task-relevant visual sequence. J. Exp. Psychol. Hum. Percept. Perform. 31, 316-327. doi: 10.1037/0096-1523.31. 2.316

Jones, D. M., Alford, D., Macken, W. J., Banbury, S. P., and Tremblay, S. (2000). Interference from degraded auditory stimuli: linear effects of changing-state in the irrelevant sequence. J. Acoust. Soc. Am. 108, 1082-1088. doi: 10.1121/1. 1288412

Jones, D. M., Hughes, R. W., and Macken, W. J. (2010). Auditory distraction and serial memory: the avoidable and the ineluctable. Noise Health 12, 201-209. doi: 10.4103/1463-1741.70497

Jones, D. M., and Macken, W. J. (1993). Irrelevant tones produce an irrelevant speech effect: Implications for phonological coding in working memory. J. Exp. Psychol. Learn. Mem. Cogn. 19, 369-381. doi: 10.1037/0278-7393.19.2.369

Jones, D. M., and Tremblay, S. (2000). Interference in memory by process or content? A reply to Neath (2000). Psychon. Bull. Rev. 7, 550-558.

Kimura, D. (1961). Cerebral dominance and the perception of verbal stimuli. Can. J. Psychol. 15, 166-171. doi: 10.1037/h0083219

Kimura, D. (1967). Functional asymmetry of the brain in dichotic listening. Cortex 3, 163-178. doi: 10.1016/S0010-9452(67)80010-8

Marsh, J. E., Hughes, R. W., and Jones, D. M. (2008). Auditory distraction in semantic memory: A process-based approach. J. Mem. Lang. 58, 682-700. doi: 10.1016/j.jml.2007.05.002

Marsh, J. E., Hughes, R. W., and Jones, D. M. (2009). Interference by process, not content, determines semantic auditory distraction. Cognition 110, 23-38. doi: 10.1016/j.cognition.2008.08.003

Marshuetz, C. (2005). Order information in working memory: An integrative review of evidence from brain and behavior. Psychol. Bull. 131, 323-339. doi: 10.1037/0033-2909.131.3.323

Neath, I. (2000). Modeling the effects of irrelevant speech on memory. Psychon. Bull. Rev. 7, 403-423.

Poeppel, D., Guillemin, A., Thompson, J., Fritz, J., Bavelier, D., and Braun, A. (2004). Auditory lexical decision, categorical perception, and FM direction discrimination differentially engage left and right auditory cortex. Neuropsychologia 42, 183-200. doi: 10.1016/j.neuropsychologia.2003.07.010

Salamé, P., and Baddeley, A. D. (1982). Disruption of short-term memory by unattended speech: implications for the structure of working memory. J. Verb. Learn. Verb. Behav. 21, 150-164. doi: 10.1016/S0022-5371(82) 90521-7

Salamé, P., and Baddeley, A. D. (1986). Phonological factors in STM: similarity and the unattended speech effect. Bull. Psychon. Soc. 24, 263-265.

Smith, E. E., Jonides, J., and Koeppe, R. A. (1996). Dissociating verbal and spatial working memory using PET. Cereb. Cortex 6, 11-20. doi: 10.1093/cercor/ 6.1.11

Sörqvist, P. (2010). High working memory capacity attenuates the deviation effect but not the changing-state effect: further support for the duplexmechanism account of auditory distraction. Mem. Cognit. 38, 651-658. doi: 10.3758/MC.38.5.651

Sörqvist, P., Marsh, J. E., and Jahncke, H. (2010). Hemispheric asymmetries in auditory distraction. Brain Cogn. 74, 79-87. doi: 10.1016/j.bandc.2010. 06.007

Sörqvist, P., Marsh, J. E., and Nöstl, A. (2013). High working memory capacity does not always attenuate distraction: Bayesian evidence in support of the null hypothesis. Psychon. Bull. Rev. 20, 897-904. doi: 10.3758/s13423-013$0419-\mathrm{y}$

Studdert-Kennedy, M., and Shankweiler, D. (1970). Hemispheric specialization for speech perception. J. Acoust. Soc. Am. 48, 579-594. doi: 10.1121/1.1912174

Tervaniemi, M., and Hugdahl, K. (2003). Lateralization of auditory-cortex functions. Brain Res. Rev. 43, 231-246. doi: 10.1016/j.brainresrev.2003. 08.004

Zahn, R., Huber, W., Drews, E., Erberich, S., Krings, T., Willmes, K., et al. (2000). Hemispheric lateralization at different levels of human auditory word processing: 
a functional magnetic resonance imaging study. Neurosci. Lett. 287, 195-198. doi: 10.1016/S0304-3940(00)01160-5

Zatorre, R. J., Evans, A. C., and Meyer, E. (1994). Neural mechanisms underlying melodic perception and memory for pitch. J. Neurosci. 14, 1908-1919.

Conflict of Interest Statement: The authors declare that the research was conducted in the absence of any commercial or financial relationships that could be construed as a potential conflict of interest.

Received: 14 October 2013; accepted: 09 December 2013; published online: 24 December 2013
Citation: Marsh JE, Pilgrim LK and Sörqvist P (2013) Hemispheric specialization in selective attention and short-term memory: a fine-coarse model of left-and right-ear disadvantages. Front. Psychol. 4:976. doi: 10.3389/fpsyg.2013.00976

This article was submitted to Cognition, a section of the journal Frontiers in Psychology. Copyright (c) 2013 Marsh, Pilgrim and Sörqvist. This is an open-access article distributed under the terms of the Creative Commons Attribution License (CC BY). The use, distribution or reproduction in other forums is permitted, provided the original author(s) or licensor are credited and that the original publication in this journal is cited, in accordance with accepted academic practice. No use, distribution or reproduction is permitted which does not comply with these terms. 